\title{
Complications of the surgical treatment of esophageal cancer and microbiological analysis of the respiratory tract
}

\author{
Radek Vrba ${ }^{a}$, Cestmir Neorala ${ }^{a}$ Katherine Vomackova ${ }^{a}$, David Vrana ${ }^{b}$, Bohuslav Melichar ${ }^{b}$, Lucie Lubuskac, \\ Yvona Loveckova ${ }^{\mathrm{d}}$, Rene Aujesky ${ }^{\mathrm{a}}$
}

\begin{abstract}
Aim. The aim of this study was to reduce the severe respiratory complications of esophageal cancer surgery often leading to death.

Methods. Two groups of patients operated on for esophageal cancer were evaluated in this retrospective analysis. The first group was operated between 2006-2011, prior to the implementation of preoperative microbiological examination while the second group had surgery between 2012-2017 after implementation of this examination.

Results. In total, 260 patients, 220 males and 40 females underwent esophagectomy. Between 2006-2011, 113 (87.6\%) males and 16 (12.4\%) females and between 2012-2017, esophagectomy was performed in 107 (81.7\%) males and 24 (18.3\%) females. In the first cohort, 10 patients died due to respiratory complications. The 30-day mortality was $6.9 \%$ and 90 -day was $9.3 \%$. In the second cohort, 4 patients died from respiratory complications. The 30-day mortality was $1.5 \%$ and 90 -day mortality was $3.1 \%$. With regard to the incidence of respiratory complications $(P=0.014)$, these occurred more frequently in patients with sputum collection, however, severe respiratory complications were more often observed in patients without sputum collection. Significantly fewer patients died $(P=0.036)$ in the group with sputum collection. The incidence of respiratory complications was very significantly higher in the patients who died $(P<0.0001)$. Conclusion. The incidence of severe respiratory complications (causing death) may be reduced by identifying clinically silent respiratory tract infections.
\end{abstract}

Key words: esophageal cancer, respiratory complications, mortality, morbidity, preoperative examination

Received: January 21, 2019; Revised: April 19, 2019; Accepted: August 12, 2019; Available online: September 13, 2019 https://doi.org/10.5507/bp.2019.040

(c) 2020 The Authors; https://creativecommons.org/licenses/by/4.0/

${ }^{a}$ Department of Surgery, Faculty of Medicine and Dentistry, Palacky University Olomouc, Czech Republic ${ }^{b}$ Department of Oncology, Faculty of Medicine and Dentistry, Palacky University Olomouc, Czech Republic 'Department of Surgical Intensive Care, Faculty of Medicine and Dentistry, Palacky University Olomouc, Czech Republic ${ }^{d}$ Department of Microbiology, Faculty of Medicine and Dentistry, Palacky University Olomouc, Czech Republic Corresponding author: Rene Aujesky, e-mail: Rene.Aujesky@fnol.cz

\section{INTRODUCTION}

Postoperative respiratory complications significantly affect the morbidity and mortality of patients after esophageal cancer surgery. The incidence in the postoperative period following classical, as well as minimally invasive esophagectomy ranges from $19.3-44.4 \%$ (ref. ${ }^{1,2}$ ). Less severe complications, including fluidothorax, pneumothorax, pneumonia, and chylothorax, also affect patient morbidity. In contrast, severe complications like ARDS (Acute respiratory distress syndrome) and respiratory failure are life-threatening in patients following esophagecto$\mathrm{my}^{3}$. The incidence of respiratory complications recorded in our institution correlates with the numbers reported in the literature, and respiratory complications were also the dominating cause of death in patients following esophagectomy for esophageal cancer. Since 2012, we have included a sputum culture for microbiological analysis of the upper and lower respiratory tract in the preoperative examination of patients with esophageal cancer in order to reduce severe respiratory complications. The purpose of this examination was to identify clinically silent infec- tion or colonisation of the respiratory tract mucosa by potential pathogens ${ }^{4}$. In cases of positive findings, the surgery was delayed or optimal prophylactic antibiotic therapy prior to the surgery was instituted ${ }^{5}$. The primary aim of the microbiological examination was to reduce the respiratory complications and reduce the mortality due to these complications.

\section{MATERIALS AND METHODS}

Retrospective analysis was performed to evaluate two cohorts of patients of similar size operated on for esophageal cancer. The first had surgery between 20062011, before systematic microbiological examinations were instituted, and the second consisted of patients treated between 2012-2017 in whom microbiological examinations were performed. The study was approved by the University Hospital Ethics Commitee. In the second cohort, a sputum culture was obtained from the patients 7 days prior to the surgery. 
The sputum samples were processed using standard methods. After homogenization, microscopic examination (Gram stain) was performed to eliminate non-purulent samples. Only samples with a significant number of leukocytes were cultivated. The selected samples were diluted followed by inoculation of the cultivation media (Columbia agar, McConkey agar and Sabouraud agar). After incubation, each sample was analyzed quantitatively. The finding of $10^{6}-10^{7}$ bacteria/mL was considered probably not an infection of the lower respiratory tract, $10^{8}$ $10^{9}$ bacteria/mL were considered probably an infection of the lower respiratory tract, and finding of $10^{10}-10^{11}$ bacteria/mL were considered an infection of the lower respiratory tract. In case a limited sample of sputum was collected, only semiquantitative analysis was performed in which the quantity of microbes was rarely found to be as high as $3+++$. If pathogens were present, the exact identification was performed using the mass spectometry Maldi-Toff (Bruker) method and the sensitivity to antimicrobial drugs was determined primarily quantitatively by the determination of the minimal inhibitory concentration (MIC). Throat swabs were inoculated into the cultivation media (Columbia agar, McConkey agar, Sabouraud agar), analysis was standardly performed after incubation of 24 and $48 \mathrm{~h}$ semiquantitatively (analysis of microbe quantity up to $3+++$ ). If pathogens were discovered, the processing was the same as with the sputum. In case of a positive microbiological finding, surgery was postponed and patients were treated by antibiotics, based on individual consultations with the antibiotic center. In cases where pathogens were found in lower quantity (up to $10^{8} / \mathrm{mL}$ ), targeted antibiotic prophylaxis was administered prior to esophagectomy based on sensitivity. Neoadjuvant chemoradiation and surgical procedures were identical in both groups of patients. Patients with early stage esophageal cancer (T1, 2, N0, M0) underwent primary surgery, patients with more advanced stage without metastatic spread, based on PET/CT (Positron emission tomography/computed tomography) findings, underwent neoadjuvant chemoradiation with subsequent surgery. Neoadjuvant therapy consisted of three cycles of the combination of 5-fluorouracil and cisplatin and radiotherapy was adminstered in fractions of $2 \mathrm{~Gy}$ to a total dose of $50 \mathrm{~Gy}$ starting with the second cycle of 5-fluorouracil and cisplatin. Amoxicillin/clavulanic acid $1.2 \mathrm{~g}$ in three daily doses was routinely administered as prophylaxis perioperatively. Patients with esophageal cancer localized aborally underwent esophagectomy, in which the resection phase was performed using a minimally invasive transhiatal approach. The esophagus with tumor was isolated to the level of the tracheal bifurcation, and a standard lymphadenectomy of the posterior mediastinum was also performed. Exstirpation of the esophagus and the reconstruction phase were performed from a midline mini-laparotomy and cervical approach. The resected stomach was used as a conduit in the majority of patients as gastroplasty. The anastomosis was constructed in two modifications completely by hand using a continuous suture in a single layer; since 2017, based on the surgeons choice, in some cases a linear stapler was used to construct the posterior circumference of the anastomosis and a continuous single layer suture was used for the anterior circumference. For tumors located in the middle or oral portion of the thoracic esophagus, a transthoracic approach was selected. We preferred a minimally invasive right-sided thoracoscopic esophagectomy for the resection phase of the surgery; only in cases of bulky tumors based on preoperative examinations (Endoultrasound or PET/ CT), a classical posterolateral right-sided thoracotomy was used. After mobilisation of the thoracic portion of the esophagus and extended mediastinal lymphadenectomy, the surgery proceeded identically to the transhiatal esophagectomy. A pyloroplasty was part of the operation in all patients. After the surgery, the patients were monitored in the intensive care unit. If the clinical condition allowed, extubation was performed within $6 \mathrm{~h}$ of the end of the operation. The patients received enteral (nutritive biluminal nasojejunal tube) and parenteral nutrition. A follow-up swallow was performed on the 7 th postoperative day to verify the sufficiency of the anastomosis and the gastroplasty or coloplasty. Patients with favourable findings were transferred to a standard care ward and gradually realimented. After full realimentation, the patients were discharged from the hospital. Statistical analysis evaluated the incidence of respiratory complications, which were divided into two groups. The first group consisted of patients with severe respiratory complications (ARDS or respiratory failure). Based on the Clavien-Dindo classification of complications, these were considered $4 \mathrm{a}$ and $4 \mathrm{~b}$. The second group included patients with less severe respiratory complications (pneumothorax, pleural effusion, pneumonia, atelectasis), based on Clavien-Dindo classification of complications these were classified 1-3. The TNM classification (The TNM Classification of Malignant Tumours) from 2009 was used for definite disease staging. Statistical analysis was used to evaluate the two groups for the following parameters: gender, type of tumor, neoadjuvant therapy, ASA classification, surgical approach (transhiatal or transthoracic), number of blood transfusions administered, respiratory complications, types of respiratory complications, anastomotic fistula, Clavien-Dindo classification, mortality. In addition, gender, tumor type, neoadjuvant therapy, ASA classification, sputum culture, surgical approach (transhiatal, transthoracic), number of blood transfusions administered, respiratory complications, types of respiratory complications, anastomotic fistula were compared between patients surviving the surgery and patients who died due to postoperative complications. Statistical software IBM SPSS Statistics version 22 was used. Fisher's exact test was used to compare qualitative parameters in patients with sputum cultures and without sputum cultures and to compare deceased and surviving patients. Quantitative parameters were compared using the Mann-Whitney U test. Normality of the data was tested using the Shapiro-Wilk test. The significance level was 0.05 . 


\section{RESULTS}

A total of 260 patients, 220 males and 40 females, underwent surgery (Table 1). Between 2006-2011, 113 (87.6\%) males and 16 (12.4\%) females, median age 59 (range 37-79) years were operated. Between 2012-9/2017, $107(81.7 \%)$ males and 24 (18.3\%) females, median age 62 (range 34-81) years were treated. In the earlier cohort, $75(58.1 \%)$ patients had adenocarcinoma, $52(40.3 \%)$ squamous cell carcinoma and $2(1.6 \%)$ had other tumor types. In the later cohort, $83(63.4 \%)$ patients had adenocarcinoma, 45 (34.4\%) had squamous cell carcinoma, and $3(2.3 \%)$ had another histological tumor type. In the earlier cohort, 100 (77.5\%) patients underwent neoadjuvant chemoradiation and in the later cohort 103 (78.6\%) patients had neoadjuvant therapy. According to the ASA (The American Society of Anesthesiologists) classification, 5 (3.9\%) patients were ASA I. , 60 (46.5\%) patients were ASA II. , and 64 (49.6\%) patients were ASA III. in the earlier cohort, while in the later cohort there were $5(3.8 \%)$ ASA I. patients, 72 (55.0\%) ASA II. patients, and 54 (41.2\%) ASA III. patients. Patients classified as ASA IV. were contraindicated for surgery. Positive sputum cultures or throat swabs (defined as containing 1 or more potential pathogens) were recorded in $36(27.5 \%)$ patients of a total 131. None of these patients displayed signs of acute respiratory infection (laboratory biomarkers of inflammation and chest X-ray were negative). Culture analysis revealed Gram-negative and Gram-positive agents (Table 5): Gram-negative agents (Pseudomonas aeruginosa, Klebsiella pneumoniae, Escherichia coli, Enterobacter cloacae, Klebsiella oxytoca, Acinetobacter ursingii, Haemophilus influenzae, Haemophilus parainfluenzae, Haemophilus haemolyticus, Neisseria meningitidis, Moraxella catarrhalis, Aggretibacter aphrophilus), Grampositive agents (Streptococcus constellatus, Streptococcus sp. beta-hemolyticus, Staphylococcus aureus MRSA, Candida albicans). If the incidence of potential pathogens in the sample was high $\left(10^{10}-10^{11} / \mathrm{mL}\right)$, the procedure was postponed in 9 cases and the patients were treated by antimicrobial therapy based on antibiograms. Subsequent follow-up sputum cultures showed no findings of potential pathogens and the patients were able to undergo surgery. In 16 patients, based on microbiological analysis of sputum cultures and throat swabs, the standard prophylactic antibiotic therapy was adjusted to targeted prophylaxis based on the given results (in cases of lower incidence of potential pathogens- $10^{8}-10^{9} / \mathrm{mL}$ ). In 11 patients with only semiquantitative sputum analysis, standard prophylaxis was administered. In 45 (34.3\%) patients, it was not possible to obtain sputum, and therefore culture analysis was performed from a throat swab. In the first group, a transhiatal laparoscopic esophagectomy with cervical anastomosis was performed in $86(66.7 \%)$ patients, in the second group in 105 (80.2\%), and transthoracic esophagectomy with cervical anastomosis in 43 (33.3\%) patients in the first group and in $26(19.8 \%)$ patients in the second group. The medial volume of blood loss during surgery was $617 \mathrm{~mL}$ in the earlier cohort and $525 \mathrm{~mL}$ in the later cohort. The median number of lymph nodes removed in
Table 1. Characteristics of patients operated for esophageal cancer from 2006-2017.

\begin{tabular}{lcc}
\hline & number & $\%$ \\
\hline Total patients & 260 & \\
$\quad$ Male & 220 & 84.6 \\
$\quad$ Female & 40 & 15.4 \\
Adenocarcinoma & 158 & 60.7 \\
Squamous cell carcinoma & 97 & 37.4 \\
Neoadjuvant therapy & 203 & 78 \\
ASA & & \\
$\quad$ I. & 10 & 3.9 \\
$\quad$ II. & 132 & 50.8 \\
$\quad$ III. & 118 & 45.3 \\
Esophagectomy & 260 & \\
$\quad$ Transhiatal & 191 & 73.4 \\
$\quad$ Transthoracic & 69 & 26.6 \\
Transfusion & 144 & 55.3 \\
Respiratory complications mild & 64 & 24.6 \\
Respiratory complications severe & 31 & 11.9 \\
Anastomotic fistula & 27 & 10.3 \\
Mortality 30 - day & 11 & 4.2 \\
Mortality 90 - day & 5 & 1.9 \\
\hline
\end{tabular}

Table 2. Results of the patient set according to Clavien-Dindo classification of complications.

\begin{tabular}{cccc}
\hline $\begin{array}{c}\text { Clavien- } \\
\text { Dindo }\end{array}$ & $\begin{array}{c}\text { Without } \\
\text { sputum culture }\end{array}$ & $\begin{array}{c}\text { With sputum } \\
\text { culture }\end{array}$ & $P$ \\
\hline 1. & $22(24.7 \%)$ & $19(15.3 \%)$ & $<0.0001$ \\
2. & $28(31.5 \%)$ & $67(54.0 \%)$ & \\
$3 . \mathrm{a}$ & $8(9 \%)$ & $18(14.5 \%)$ & \\
$3 . \mathrm{b}$ & $2(2.2 \%)$ & $9(7.3 \%)$ & \\
$4 . \mathrm{a}$ & $11(12.4 \%)$ & $5(4.0 \%)$ & \\
$4 . \mathrm{b}$ & $6(6.7 \%)$ & $2(1.6 \%)$ & \\
5. & $12(13.5 \%)$ & $4(3.2 \%)$ & \\
\hline
\end{tabular}

the earlier cohort was 12 (range 2-28), while in the later cohort the median was 11 (range 3-38). The median duration of surgery in the earlier cohort was 209 min and 212 min in the later cohort. In the earlier cohort, respiratory complications in the postoperative course were recorded in $39(30.2 \%)$ patients, with $22(17.1 \%)$ considered severe (ARDS or respiratory failure). In the later cohort, respiratory complications were observed in 56 (42.7\%) patients, including severe complications in $9(6.9 \%)$ patients. Anastomotic fistula was observed in 12 (9.3\%) patients in the earlier cohort and in $15(11.5 \%)$ patients in the later cohort. Blood transfusions during the surgery or postoperatively were administered in 76 (58.9\%) patients in the earlier cohort and to $68(51.9 \%)$ patients in the later cohort. Patients from both cohorts were staged according to the 2009 TNM classification. Pathological complete response was observed in $15(11.6 \%)$ patients in the earlier cohort and in 38 (29\%) patients in the later cohort. Postoperative complications were evaluated according to the Clavien-Dindo classification of surgical complications 
Table 3. Comparison of results between patients without sputum culture from 2006-2011 and patients with sputum culture from 2012-2017.

\begin{tabular}{lccc}
\hline & Without sputum culture & Sputum culture & $P$ \\
\hline Gender & $113(87.6 \%)$ & $107(81.7 \%)$ & 0.186 \\
Male & $16(12.4 \%)$ & $24(18.3 \%)$ & \\
Female & & & \\
Tumour type & $75(58.1 \%)$ & $83(63.4 \%)$ & \\
$\quad$ Adenocarcinoma & $52(40.3 \%)$ & $45(34.4 \%)$ & 0.602 \\
Squamous cell & $2(1.6 \%)$ & $3(2.3 \%)$ & \\
Other type & $100(77.5 \%)$ & $103(78.6 \%)$ & 0.829 \\
Neoadjuvant therapy & & & \\
ASA & $5(3.9 \%)$ & $72(5.8 \%)$ & \\
I. & $60(46.5 \%)$ & $54(41.2 \%)$ & 0.382 \\
II. & $64(49.6 \%)$ & $36(27.5 \%)$ & \\
III. & 0 & & \\
Positive sputum & & $105(80.2 \%)$ & 0.014 \\
Type of esophagectomy & & $26(19.8 \%)$ & \\
Transhiatal & $86(66.7 \%)$ & $68(51.9 \%)$ & 0.256 \\
Transthoracic & $43(33.3 \%)$ & & \\
Transfusion & $76(58.9 \%)$ & $5(6.9 \%)$ & 0.570 \\
Respiratory complications & & $15(11.5 \%)$ & 0.036 \\
Less severe & $39(30.2 \%)$ & & \\
Severe & $22(17.1 \%)$ & & \\
Anastomotic fistula & $12(9.3 \%)$ & & \\
Mortality & $12(9.3 \%)$ & & \\
\hline
\end{tabular}

(Table 2). There were $12(9.3 \%)$ deaths in the earlier cohort and $4(3.1 \%)$ in the later cohort. In the earlier cohort, 10 patients died due to respiratory complications, one patients died on the 3 rd postoperative day due to extensive myocardial infarction and one patient died due to necrosis of the conduit with anastomotic fistula. The 30-day mortality was $6.9 \%$ and 90 -day mortality was $9.3 \%$. In the later cohort, all patients died from respiratory complications, 30 -day mortality was $1.5 \%$, and 90 -day was $3.1 \%$. A significant difference between patients with sputum cultures and without sputum cultures was observed in the type of procedure $(P<0.0001)$ as there were significantly more transhiatal procedures in the later cohort. The incidence of respiratory complications $(P=0.014)$ was higher in the second cohort; however, severe respiratory complications were more frequent in the first cohort. Significantly fewer patients died $(P=0.036)$ in the cohort of patients with sputum culture (Table 3 ). The respiratory complications of those who died was much higher than those who lived $(P<0.0001)$ and those who died had a significantly higher ASA classification (Table $4 ; P=0.003$ ).

\section{DISCUSSION}

The data indicate that microbiological monitoring leading to prophylactic administration of antibiotics can significantly reduce severe respiratory complications, the principal cause of mortality after surgery for esopha- geal cancer ${ }^{4}$. Esophagectomy that canbe performed by a minimally invasive, classical, or hybrid approach remains the only curative treatment for esophageal carcinoma. Based on tumor location, a transhiatal approach without opening the thoracic cavity with anastomosis in the deep cervical space (Orringer) or a combined approach from laparotomy (laparoscopy) and thoracotomy (thoracoscopy) with anastomosis in the thoracic cavity (Iwor-Lewis esophagectomy) or anastomosis in the deep cervical space (McKeown esophagectomy) may be selected. A tubulized stomach supplied by the right gastroepiploic artery in the form of a gastroplasty is most commonly used to reconstruct the digestive tract. If it is not possible to use the stomach (usually because of tumor infiltration of the stomach or prior stomach resections that disturbe the blood supply), the colon is used in the form of a coloplasty. Only in tumors diagnosed in early stage T1a, therapeutic mucosectomy of the tumor may be performed instead of an esophagectomy. The literature still reports a fairly high morbidity rate associated with esophagectomy that ranges between 20 and 50\% (ref. ${ }^{6,7}$ ). In contrast, the mortality has significantly decresed in the past few decades and is currently reported to range from 1 to $5.8 \%$ (ref. $^{8,9}$ ). The most frequent cause of death following esophagectomy are respiratory complications, the incidence of which increases with age, chronic nicotine use, malnutrition, pulmonary dysfunction and immunodeficiency ${ }^{10,11}$. Other factors which affect the development of respiratory complications include duration of surgery 
Table 4. Statistical evaluation of surviving patients and those who died postoperatively

\begin{tabular}{|c|c|c|c|}
\hline & Surviving & Died & $P$ \\
\hline Sputum culture & $127(52.0 \%)$ & $4(25.0 \%)$ & 0.036 \\
\hline W/o sputum culture & $117(48.0 \%)$ & $12(75 \%)$ & \\
\hline Gender & & & 0.282 \\
\hline Male & $208(85.2 \%)$ & $12(75 \%)$ & \\
\hline Female & $36(14.8 \%)$ & $4(25 \%)$ & \\
\hline Type of tumour & & & 0.583 \\
\hline Adenocarcinoma & $150(61.5 \%)$ & $8(50 \%)$ & \\
\hline Squamous cell ca. & $89(36.5 \%)$ & $8(50 \%)$ & \\
\hline Other & $5(2.0 \%)$ & 0 & \\
\hline Neoadjuvant therapy & $190(77.9 \%)$ & $13(81.3 \%)$ & 1 \\
\hline W/o neoadjuvant ther. & $54(22.1 \%)$ & $3(18.8 \%)$ & \\
\hline ASA & & & 0.003 \\
\hline I. & $10(4.1 \%)$ & 0 & \\
\hline II. & $130(53.3 \%)$ & $2(12.5 \%)$ & \\
\hline III. & $104(42.6 \%)$ & $14(87.5 \%)$ & \\
\hline Positive sputum culture & $34(26.8 \%)$ & $2(50 \%)$ & 0.303 \\
\hline Negative sputum culture & $93(73.2 \%)$ & $2(50 \%)$ & \\
\hline Esophagectomy & & & 1 \\
\hline Transhiatal & $179(73.4 \%)$ & $12(75 \%)$ & \\
\hline Transthoracic & $65(26.6 \%)$ & $4(25 \%)$ & \\
\hline Transfusion & $134(54.9 \%)$ & $10(62.5 \%)$ & 0.554 \\
\hline $\mathrm{W} / \mathrm{o}$ transfusion & $110(45.1 \%)$ & $6(37.5 \%)$ & \\
\hline $\begin{array}{l}\text { Respir. complications } \\
\text { less severe }\end{array}$ & $95(36.5 \%)$ & 0 & $<0.0001$ \\
\hline $\begin{array}{l}\text { Respir. complications } \\
\text { severe }\end{array}$ & $17(7.0 \%)$ & $14(87.5 \%)$ & \\
\hline $\begin{array}{l}\text { W/o respiratory } \\
\text { complications }\end{array}$ & $132(50.8 \%)$ & $2(12.5 \%)$ & \\
\hline Anastomotic fistula & $26(10.7 \%)$ & $1(6.2 \%)$ & 1 \\
\hline $\mathrm{W} / \mathrm{o}$ fistula & $218(89.3 \%)$ & $15(93.8 \%)$ & \\
\hline
\end{tabular}

Table 5. Summary of isolated potential microbial pathogens by cultivation of sputum (in samples of 5 patients were isolated more pathogens than one species).

\begin{tabular}{llc}
\hline Isolated strain & Gram stain & $\begin{array}{c}\text { Number of isolated strains } \\
(\%)\end{array}$ \\
\hline Streptococcus constellatus & Gram-positive & $4(9.7)$ \\
Streptococcus sp. beta-hemolyticus & Gram-positive & $4(9.7)$ \\
Staphylococcus aureus MRSA & Gram-positive & $4(9.7)$ \\
Candida albicans & Gram-positive & $3(7.3)$ \\
Pseudomonas aeruginosa & Gram-negative & $2(4.8)$ \\
Klebsiella pneumoniae & Gram-negative & $4(9.7)$ \\
Escherichia coli & Gram-negative & $3(7.3)$ \\
Enterobacter cloacae & Gram-negative & $2(4.8)$ \\
Klebsiella oxytoca & Gram-negative & $2(4.8)$ \\
Acinetobacter ursingii & Gram-negative & $1(2.4)$ \\
Haemophilus influenzae & Gram-negative & $4(9.7)$ \\
Haemophilus parainfluenzae & Gram-negative & $2(4.8)$ \\
Haemophilus haemolyticus & Gram-negative & $2(4.8)$ \\
Neisseria meningitidis & Gram-negative & $1(2.4)$ \\
Moraxella catarrhalis & Gram-negative & $2(4.8)$ \\
Aggretibacter aphrophilus & Gram-negative & $1(2.4)$ \\
\hline
\end{tabular}


and perioperative blood loss, decreased forced expiratory volume (FEV) 1 on spirometry, neoadjuvant oncological therapy and the incidence of anastomotic fistula ${ }^{12,13}$. The incidence of postoperative respiratory complications may be influenced preoperatively by improving lung function, careful administration of anesthesia during the surgery and an early extubation of the patients ${ }^{14,15}$. Uchihara et al. reported the incidence of respiratory complications following minimally invasive esophagectomy of $17.9 \%$. In that cohort, univariate analysis established risk factors including smoking, neoadjuvant therapy, advanced disease stage (stage III. IV.), and intraoperative blood loss above $600 \mathrm{~g}$ (ref. ${ }^{16}$ ). Ryan et al. report that the incidence of hypoalbuminemia on the first postoperative day is associated with a higher morbidity, including respiratory complications ${ }^{17}$. In general, the incidence of respiratory complications is considered a prognostically negative factor of long-term results in patients following esophagectomy ${ }^{18,19}$. Low et al. reported an international consensus of standardization of respirátory complications associated with esophagectomy that included pneumonia, pleural effusions requiring drainage, pneumothorax requiring therapy, atelectasis requiring bronchoscopy, acute aspiration, tracheobronchial injury, retaining the chest drain longer than 10 days due to air leak, respiratory failure requiring reintubation, and ARDS ( ref. $^{3}$ ). Respiratory failure and ARDS have the greatest impact on mortality due to respiratory complications, with mortality ranging between $30-60 \%$ (ref. $^{20}$ ). A number of reports tried to define approaches to decrease the postoperative morbidity and select the ideal surgical approach (MIE, classical esophagectomy or hybrid type of operation). Yun et al. reported less severe respiratory complications after MIE (Miniinvasive esophagestomy) (15.7\%) compared to classical esophagectomy (42.9\%) (ref. ${ }^{21}$ ). Moon et al. observed a longer operating time when performing thoracoscopical esophagectomy compared to a classical approach, but significantly less respiratory complications ( $9.5 \%$ vs. $40.5 \%$, $P=0.004$ ) ( ref. $^{22}$ ). Growing experience with minimally invasive esophagectomy has been associated with a decrease in postoperative respiratory complications ${ }^{23-25}$. Hayami et al. described postoperative complications in $60 \%$ of patients, respiratory complications in $32.9 \%$ and anastomotic fistula in $12.9 \%$ (ref. $^{26}$ ). The incidence of other complications, e.g. anastomotic dehiscence or conduit necrosis, are reported in individual patient series in significantly lower numbers than respiratory complications. In contrast to respiratory complications, less severe complications practically do not affect the mortality if properly treated. In the present cohort of patients, the dominant cause of death were respiratory complications (in 14 patients). In the earlier cohort, 10 patients died of respiratory complications with two death of other causes. In the later cohort, all four postoperative death were caused by respiratory complications. Based on statistical analysis of the results of both cohorts, we determined that there was a lower number of all respiratory complications in the earlier cohort, but there were significantly fewer severe respiratory complications and deaths due to respiratory complications in the later cohort. Cardiac complications during the postoperative course occur in up to $10 \%$ of patients ${ }^{27,28}$, but in the present series only one patient died due to extensive myocardial infarction. Necrosis of the conduit may be regarded as the most severe surgical complication, which more often affects a coloplasty (13\%) compared to a gastroplasty $(0.5 \%)$. This complication is treated by exstirpation of the conduit, creating a cervical esophagostomy and nutritive jejunostomy. If the patient overcomes this life-threatening complication, secondary reconstruction of the gastrointestinal tract is performed. Ramage et al. reported necrosis of the gastroplasty in 4 patients $(2.6 \%)$. Treatment was successful in two of these cases (in one patient reconstruction was performed using the colon, the second patient refused reconstruction) $\left(\right.$ ref. $\left.^{29}\right)$. We observed necrosis of the gastroplasty in one patient and the patient died as a result of this complication. Anastomotic dehiscence reported in the literature ranges from $5 \%$ to $20 \%$ (ref. $^{30,31}$ ). Risk factors include age, male gender, smoking, alcohol abuse, ASA score, prolonged operation time, low serum albumin, intraoperative blood loss, diabetes mellitus, renal failure and cardiovascular disease $\mathrm{e}^{32,33}$. Based on the international consensus evaluating complications associated with esophagectomy, three types of dehiscence have been described. Type I. local defect observed on swallow without change in therapy or only dietary measures. Type II. local defect not requiring surgical intervention, but requiring radiological intervention (stent implantation and/or drainage). Type III. requires surgical intervention ${ }^{3}$. In the present series, all types of anastomotic dehiscence according to the classification defined by Low et al. were observed ${ }^{3}$. If the thoracic duct is partially or completely transected, chylothorax may be observed. If confirmed, chylothorax is primarily treated conservatively. If unsucessful, if there is complete transection, it is necessary to treat it surgically by ligating the thoracic duct. If left untreated, chylothorax may lead to malnutrition and immunodeficiency, and represent a serious condition with a patient mortality of up to $10 \%$ (ref. ${ }^{34}$ ). Paralysis of the left reccurent laryngeal nerve in patients operated on using the cervical approach is reported in up to $14 \%$ of patients ${ }^{35}$. There is a consensus that is based on the analysis of postoperative results that surgery for esophageal cancer should be performed in high volume centres ${ }^{36}$, however despite this effort and intensive research in this field ${ }^{37-39}$ the general outcome of the treatment remains unsatisfactory.

The present retrospective analysis has some obvious limitations. Importantly, patients treated in two consecutive periods were compared and the differences observed could have been caused by other factors, e.g. different epidemiological situation. However, the incidence of respiratory infections of any severity was higher in the later cohort, while severe respiratory complications were markedly lower, indicating that the intervention, i.e. microbiological monitoring and antibiotic therapy, are responsible for lowering the severe complication rate and mortality. 


\section{CONCLUSION}

In conclusion, respiratory complications cannot be completely eliminated after esophagectomy for esophageal cancer. In the present patient series, we were able to reduce the number of severe respiratory complications and decrease mortality due to these complications. One approach to decrease respiratory complications in the postoperative course is to identify clinically silent infections or colonization of the respiratory tract prior to surgery and treat the patient with appropriate antimicrobial therapy or adjust the antimicrobial prophylaxis. Further prospective studies are necessary to confirm these results.

Author contributions: RV, CN, RA, LL, YL: patient selection and management; RV, DV, BM, KV: manuscript writing; RV, KV: statistical analysis.

Conflict of interest statement: The authors state that there are no conflicts of interest regarding the publication of this article.

\section{REFERENCES}

1. Dumont P, Wihlm JM, Hentz JG, Roeslin N, Lion R, Morand G. Respiratory complications after surgical treatment of esophageal cancer A study of 309 patients according to the type of resection. Eur J Cardioithorax Surg 1995;9:539-43.

2. Ferguson MK, Durkin AE. Preoperative prediction of the risk of pulmonary complications after esophagectomy for cancer. J Thorax Cardiovasc Surg 2002;123:661-9.

3. Low DE, Alderson D, Cecconello I, Chang AC, Darling GE, D'Journo XB, Griffin SM, Hölscher AH, Hofstetter WL, Jobe BA, Kitagawa Y, Kucharczuk JC, Law SY, Lerut TE, Maynard N, Pera M, Peters JH, Pramesh CS, Reynolds JV, Smithers BM, van Lanschot JJ. International consensus on standartization of data collection for complications associated with esophagectomy. Annals of Surgery 2015;262(2):28694.

4. Kosumi K, Baba Y, Yamashita K, Ishimoto T, Nakamura K, Ohuchi M, Kiyozumi Y, Izumi D, Tokunaga R, Harada K, Shigaki H, Kurashige J, Iwatsuki M, Sakamoto Y, Yoshida N, Watanabe M, Baba H. Monitoring sputum culture in resected esophageal cancer patients with preoperative treatment. Dis Esophagus 2017;30(12):1-9.

5. Horaková M, Lubušká L, Kolář K, Vrba R, Neoral Č, Zapletalová J.Individualized prophylaxis in patients with esophageal replacement for cancer. Surgical Infections 2015;16:513-7.

6. Wlodarczyk J, Kużdżał J. Stenting as a palliative method in the management of advanced squamous cell carcinoma of the oesophagus and gastro-oesophageal junction. Videosurgery Miniinv 2016;11(1):1-8.

7.Zhang Z, Zhang H. Impact of neoadjuvant chemotherapy and chemoradiotherapy on postoperative cardiopulmonary complications in patients with esophageal cancer. Dis Esophagus 2017;30(4):1-7.

8.Ott K, Bader FG, Lordick F, Feith M, Bartels H, Siewert JR. Surgical factors influence the outcome after Ivor-Lewis esophagectomy with intrathoracic anastomosis for adenocarcinoma of the esophagogastric junction: a consecutive series of 240 patient at an experienced center. Ann Surg Oncol 2009;16:1017-25.

9.Atkins BZ, Shan AS, Kelley A, Hutcheson KA, Mangum JH, Pappas TN Harpole DH, Dámico TA. Reducing hospital morbidity and mortality following esophagectomy. Ann Thorac Surg 2004;78:1170-6.

10. Dahn D, Martell J, Vorwerk H, Hess C F, Becker H, Jung K, Hilgers R, Wolff H A, Hermann R M, Christiansen $\mathrm{H}$. Influence of irradiated lung volumes on perioperative morbidity and mortality in patients after neoadjuvant radiochemotherapy for esophageal cancer. In J Radiat Oncol Biol Phys 2010;1:44-52.

11. Xing $X Z$, Gao $Y$, Wang $H J$, Qu SN, Huang $C L$, Zhang $H$, Wang $H$, Yang $\mathrm{QH}$. Assessment of a predictive score for pulmonary complica- tions in cancer patients after esophagectomy. World J Emerg Med. 2016;7(1):44-9.

12. Gao $Y$, Wang HJ, Qu SN, Huang $C L$, Zhang $H$, Wang $H$, Yang QH. Assessment of a predictive score for pulmonary complications in cancer patients after esophagectomy. World J Emerg Med 2016;7(1):44-9.

13. Tandon S, Batchelor A, Bullock R, Gascoigne A, Griffin M, Hayes N, Hing J, Shaw I, Warnell I, Baudouin SV. Peri-operative risk factor for acute lung injury after elative esophagectomy. Br J Anaesth 2001;86:633-8.

14. Bartels H, Stein H J, Siewert J R. Risk analysis in esophageal surgery. Resent Result Cancer Res 2000;155:89-96.

15. Dumont P, Wihlm J M, Hentz J G, Roeslin N, Lion R., Morand G. Respiratory comlications after surgical treatment of esophageal cancer A study of 309 patients according to the type of resection. Eur J Cardioithorax Surg 1995;9:539-43.

16. Uchihara T, Yoshida N, Baba Y, Yagi T, Toihata T, Oda E, Kuroda D, Eto T, Ohuchi M, Nakamura K, Sawayama H, Kinoshita K, Iwatsuki M, Ishimoto T, Sakamoto Y, Baba H. Risk factors for pulmonary morbidities after minimally invasive esophagectomy for esophageal cancer. Surg Endosc 2018;32(6):2852-8

17. Ryan AM, Hearty A, Prichard RS, Cunningham A, Rowley SP, Reynolds JV. Association of hypoalbuminemia on the first postoperative day and complications following esophagectomy. J Gastrointest Surg 2007;11:1355-60.

18. Baba Y, Yoshida N, Shigaki H, Iwatsuki M, Miyamoto Y, Sakamoto $\mathrm{Y}$, Watanabe M, Baba H. Prognostic Impact of Postoperative Complications in 502 Patients With Surgically Resected Esophageal Squamous Cell Carcinoma: A Retrospective Single-institution Study. Ann Surg 2016;264(2):305-11.

19. Lerut T, Moons J, Coosemans W, Van Raemdonck D, De Leyn P, Decaluwé H, Decker G, Nafteux P. Postoperative complications after transthoracic esophagectomy for cancer of the esophagus and gastroesophageal junction are correlated with early cancer recurrence: role of systematic grading of complications using the modified Clavien classification. Ann Surg 2009;25:798-807.

20. Wan J, Che Y, Kang N, Zhang R. Surgical Method, Postoperative Complications, and Gastrointestinal Motility of Thoraco-Laparoscopy 3-Field Esophagectomy in Treatment of Esophageal Cancer.Med SciMonit. 2016 Jun 16; 22 :2056-65.

21. Yun JS, Na KJ, Song SY, Kim S, Jeong IS, Oh SG. Comparison of perioperative outcomes following hybrid minimally invasive versus open Ivor Lewis esophagectomy for esophageal cancer. J Thorac Dis 2017;9(9):3097-104.

22. Moon DH, Lee JM, Jeon JH, Yang HC, Kim MS. Clinical outcomes of video-assisted thoracoscopic surgery esophagectomy for esophageal cancer: a propensity score-matched analysis. J Thorac Dis 2017;9(9):3005-12.

23. Lerut T. Uniportal video-assisted thoracoscopic surgery in esophageal diseases: an introduction. J Vis Surg 2017;3:182.

24. Nafteux P, Moons J, Coosemans W, Decaluwé H, Decker G, De Leyn $P$, Van Raemdonck D, Lerut T. Minimally invasive oesophagectomy: a valuable alternative to open oesophagectomy for the treatment of early oesophageal and gastro-oesophageal junction carcinoma. Eur J Cardiothorac Surg 2011;40:1455-63.

25. Biere SS, van Berge Henegouwen MI, Maas KW, Bonavina L, Rosman C, Garcia JR, Gisbertz SS, Klinkenbijl JH, Hollmann MW, de Lange ES, Bonjer HJ, van der Peet DL, Cuesta MA. Minimally invasive versus open oesophagectomy for patients with oesophageal cancer: a multicentre, open-label, randomised controlled trial. Lancet 2012;379:1887-92.

26. Hayami M, Watanabe M, Ishizuka N, Mine S, Imamura Y, Okamura A, Kurogochi T, Yamashita K. Prognostic impact of postoperative pulmonary complications following salvage esophagectomy after definitive chemoradiotherapy. J Surg Oncol 2018;117(6):1251-9.

27. Luketich JD, Alvelo-Rivera M, Buenaventura PO, Christie NA, McCaughan JS, Litle VR, Schauer PR, Close JM, Fernando HC. Minimally invasive esophaectomy: outcomes in 222 patients. Ann Surg 2003;238(4):486-94.

28. Whooly B P, Law S, Murthy S C, Alexandrou A, Wong J. Analysis of reduced death and complication rates after esophageal resection Ann Surg 2001;233:338-44.

29. Ramage L, Deguara J, Davies A, Hamouda A, Tsigritis K, Forshaw $\mathrm{M}$, Botha AJ. Gastric tube necrosis following minimally invasive 
oesophagectomy is a learning curve issue.Ann R Coll Surg Engl 2013;95(5):329-34

30. Sunpaweravong S, Ruangsin S, Laohawiriyakamol S, Mahattanobon S,Geater A. Prediction of major postoperative complications and survival for locally advanced esophageal carcinoma patients. Asian J Surg 2012;35:104-9.

31. Haga Y, Wada Y, Takeuchi H, Ikejiri K, Ikenaga M. Prediction of anastomotic leak and its prognosis in digestive surgery. World J Surg 2011;35: 716-22.

32. Van Daele E, Van de Putte D, Ceelen W, Van Nieuwenhove Y, Pattyn P. Risk factors and consequences of anastomotic leakage after Ivor Lewis oesophagectomy. Interact Cardiovasc Thorac Surg 2016;22(1):32-7.

33. Kassis E, Kosinski A, Ross P Jr, Koppens K, Donahue J, Daniel V. Predictors of anastomotic leak after esophagectomy: an analysis of the society of thoracic surgeons general thoracic database. Ann Thorac Surg 2013;96: 1919-26.

34. Nishihira T, Suzuki T, Mori S. A prospective randomized trial of extended cervical and superior mediastinal lymphadenectomy for carcinoma of the thoracic esophagus. American J Surg 1998; 175: 47-51

35. Pennathur A, Awais O, Luketich JD. Technique of minimally invasive Ivor Lewis esophagectomy. Ann Thorac Surg 2010;89:S2:159-62.

36. Tapias LF, Morse CR. Minimally invasive Ivor Lewis esophagectomy: description of a learning curve. J Am Coll Surg 2014;218:1130-40.

37. Vrana D, Matzenauer M, Aujesky R, Vrba R, Neoral C, Melichar B, Souček P. Potential Predictive Role of MicroRNAs in the Neoadjuvant Treatment of Esophageal Cancer. Anticancer Res 2017;37(2):403-12. Review.

38. Vrana D, Hlavac V, Brynychova V, Vaclavikova R, Neoral C, Vrba J, Aujesky R, Matzenauer M, Melichar B, Soucek P. ABC Transporters and Their Role in the Neoadjuvant Treatment of Esophageal Cancer. Int J Mol Sci 2018;19(3). pii: E868. Review.

39. Vrána D, Matzenauer M, Neoral Č, Aujeský R, Vrba R, Melichar B, Rušarová $N$, Bartoušková $M$, Jankowski J. From Tumor Immunology to Immunotherapy in Gastric and Esophageal Cancer. Int J Mol Sci 2018;20(1). pii: E13. Review. 